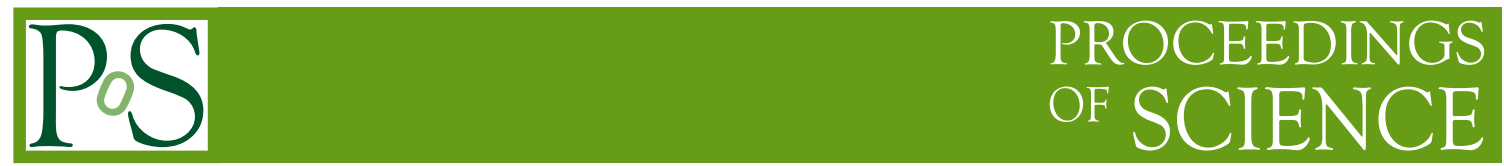

\title{
Particle identification at LHC: Alice and LHCb
}

\author{
Frederic Machefert ${ }^{* \dagger}$ \\ LAL, Université Paris-Sud, CNRS/IN2P3, Orsay, France \\ E-mail: frederic.machefertein2p3.fr
}

The Alice and LHCb collaborations equipped their detectors with many technologies to identify particles in a large momentum range, both in the barrel and the forward regions. The methods used are described and some details on the hardware implementation are given. A few physics results illustrate the performances obtained by the two detectors.

14th International Conference on B-Physics at Hadron Machines April 8-12, 2013

Bologna, Italy

\footnotetext{
*Speaker.

${ }^{\dagger}$ On behalf of the LHCb collaboration.
} 


\section{Introduction}

The Alice and LHCb detectors at CERN are the two large dedicated experiments on the ring of the LHC accelerator. Alice, A Large Ion Collider Experiment, is a dedicated heavy-ion detector which studies the physics of strongly interacting matter and the properties of the new phase of matter expected from QCD, the quark gluon plasma (QGP) $[1,2]$. The LHCb detector aims at better understanding $\mathrm{CP}$ violation and at searching for new physics through rare decay studies in the beauty sector and in charmed meson physics $[3,4]$.
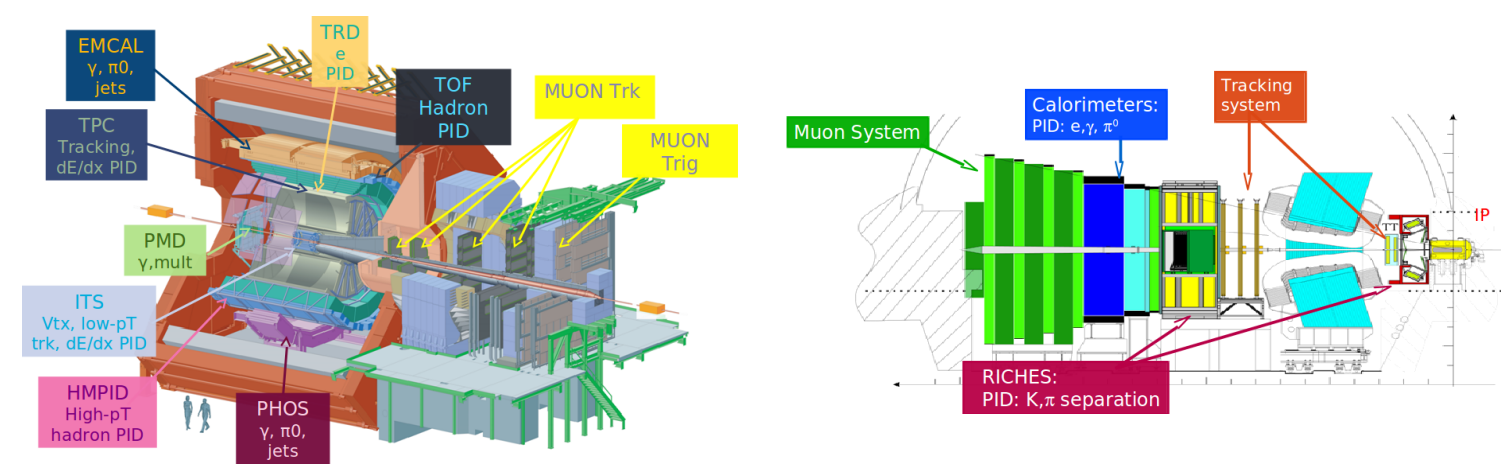

Figure 1: The geometry of the Alice (left) and LHCb (right) detectors. The sub-detectors contributing to the particle identification and described in this paper are indicated.

Both detectors require the measurement of many observables like exclusive production rates, the energy, position or angular distributions of specific types of particles. Alice characterizes the QGP studying identified particle production, correlations and flow, in particular charmed and beauty hadrons or charmonium and bottomonium. The LHCb collaboration exclusively reconstructs a large variety of $B$ and $D$ meson decay channels. Hence, the two collaborations designed many complementary and complex sub-detectors using most of the known techniques providing particle identification information. In addition, they may require such information to be available at an early stage of the trigger processing. This imposes drastic constraints on the response of the sub-detectors identifying the particles.

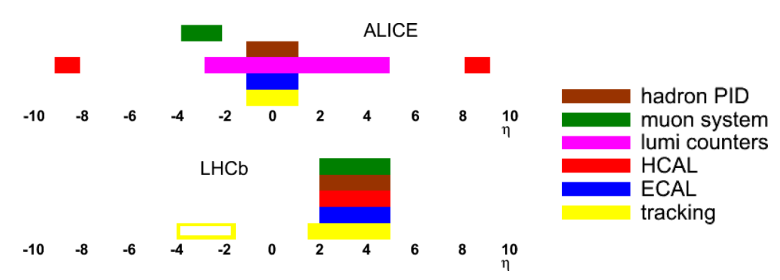

Figure 2: Rapidity regions covered by the Alice (top) and LHCb (bottom) experiments and by some of the sub-detectors used for particle identification.

The geometries of the Alice and LHCb detectors (Fig. 1) are driven by the physics goals of the two collaborations and are consequently very different. Alice looks for particles in an acceptance 
that covers both the barrel and the forward region while $\mathrm{LHCb}$ is a forward spectrometer (Fig. 2 shows the rapidity regions mapped by the two experiments). Hence, the momentum of the particles to be identified is often softer in the former than in the latter. The identification techniques used reflect those differences. The next section describes the methods used by Alice and LHCb following the path of the particles.

\section{Particle identification methods used by Alice and LHCb}

\subsection{Energy loss measurement}

Two Alice sub-detectors use the energy loss in order to classify particles. The first one is the inner tracker system (ITS) [5]. It is made of 6 detection layers based on 3 different technologies: 2 layers of silicon pixels (SPD, $3.9 \mathrm{~cm}$ away from the beam), 2 of silicon drift detectors (SDD) and 2 outermost layers of silicon strips (SSD). This system is used for the tracking and the primary vertex localization. In addition, the SDD and SSD measure the charge collected, providing an estimation of the $d E / d x$ with a resolution of $\sim 10-15 \%$ (Fig. 3). Identification of low momentum particles (down to a few hundreds of $\mathrm{MeV} / c$ ) is possible, $K-p$ and $\pi-K$ separation being achievable up to $1 \mathrm{GeV} / c$ and $450 \mathrm{MeV} / c$, respectively.

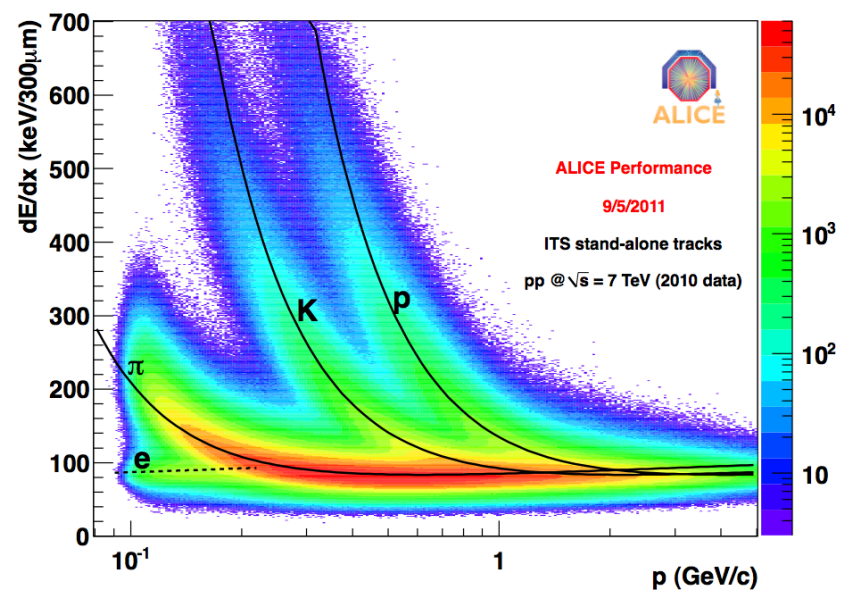

Figure 3: $d E / d x$ measured in the ITS as a function of the particle momentum.

The large time projection chamber (TPC) [6] of Alice is $5 \mathrm{~m}$ long with an inner and outer radius of 0.85 and $2.5 \mathrm{~m}$, respectively (Fig. 4, left). It is filled with $92 \mathrm{~m}^{3}$ of gas $\left(\mathrm{Ne} / \mathrm{CO}_{2} / \mathrm{N}_{2}\right)$ leading to a drift time of $92 \mu \mathrm{s}$ and read out by $\sim 560000$ pads. The $d E / d x$ measurement of the TPC extends drastically the ITS particle classification, and at larger momentum, there is the possibility of particle separation in the relativistic rise region, as can be seen from Fig. 4 (right).

\subsection{Transition radiation}

The transition radiation detector (TRD) [7] of Alice is made of 6 layers, made each of a radiator, a drift space, anode wires and cathode pads (Fig. 5, left). At the energies reached at the LHC, only electrons significantly emit transition radiation photons when they traverse boundaries 

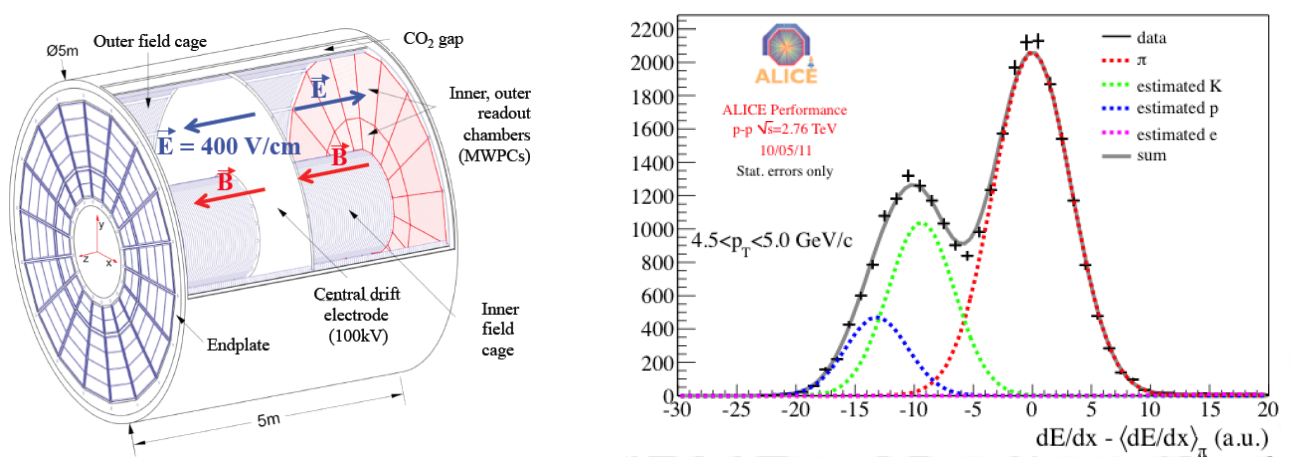

Figure 4: Schematics of the Alice large TPC (left) and $\pi, K$, proton, electron $d E / d x$ with respect to average $\pi d E / d x$ superimposed with data points.

between media of different refraction indices. Those photons produce a late pulse with respect to the signal of the charged incoming particle itself, as can be seen on Fig. 5 (right). From the signal shape, a rejection factor of $\sim 23$ is obtained on pions keeping an electron efficiency of $90 \%$ and for a momentum of $\sim 2 \mathrm{GeV} / c$.
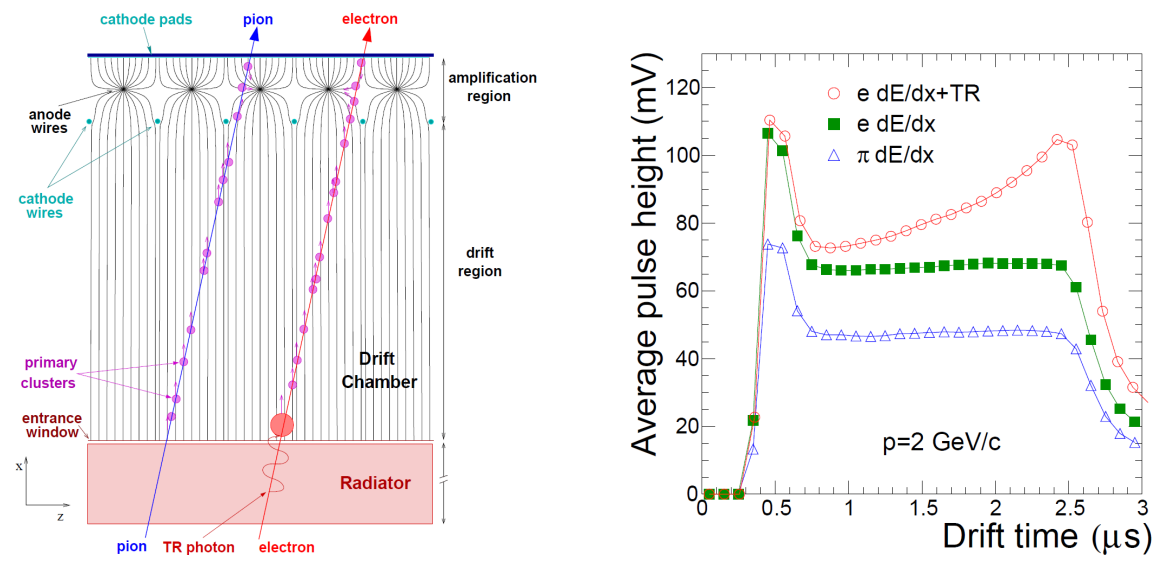

Figure 5: Drawing of a transition radiation detector layer (left) and comparison of the signal seen by the detector with and without TRD for electrons and $\pi$. The after-pulse for electrons with TRD is clearly visible for a drift time of $\sim 2.5 \mu \mathrm{s}$

The information of the ITS, TPC and TRD systems is combined using statistical methods based on maximum likelihood estimations or neural networks in order to achieve an even better rejection.

\subsection{Time of flight}

The last tool used by Alice to classify low or intermediate momentum particles is the time of flight detector (TOF) [8] which is made of multigap resistive plate chambers surrounding the barrel of the experiment, $3.7 \mathrm{~m}$ away from the beam and providing a full $\phi$ coverage with $|\eta|<0.9$. The intrinsic resolution of the TOF is $\sim 40 \mathrm{ps}$ with an overall Alice time resolution of $\sim 80 \mathrm{ps}$ with 
respect to the interaction time. The determination of the mass from the time and the momentum measurement obtained from the tracker permits to get an excellent separation up to 2 and $4 \mathrm{GeV} / c$ in transverse momentum $\left(p_{\mathrm{T}}\right)$ for $K / \pi$ and $K /$ proton, respectively.

\subsection{Cherenkov detectors}

In the forward region, the momentum of the incoming particles is larger and new methods are used both by Alice and LHCb to identify particle species. Ring Imaging Cherenkov detectors (RICH) rely on the light cones produced by charged particles travelling faster than light in a medium of index larger than 1 . LHCb uses intensively this technique with two such RICH [9]. The detector closer to the interaction point contains two Cherenkov media, aerogel and $C_{4} F_{10}$, and is efficient up to a few tens of $\mathrm{GeV} / c$ (Fig. 6, left, shows the geometry of the first LHCb RICH, RICH-1). The second RICH (RICH-2) has a smaller angular aperture, focussing towards the higher rapidity ranges and thus the larger momenta. It contains a single radiator composed of $C F_{4}$, which provides a good classification up to $\sim 100 \mathrm{GeV} / c$. In both cases, the light produced is detected by hybrid photo-detectors (HPD) located outside the LHCb acceptance, the light being reflected and extracted with both flat and spherical mirrors. The opening angle of the light cone is reconstructed and combined with tracker momentum information to determine the mass of the incoming particle. The typical angular resolution needed to separate electron, muon, $\pi, K$ and proton mass hypothesis at such high energies is 1.62 and 0.68 mrad respectively for RICH-1 and RICH-2 (see Fig. 6, right).
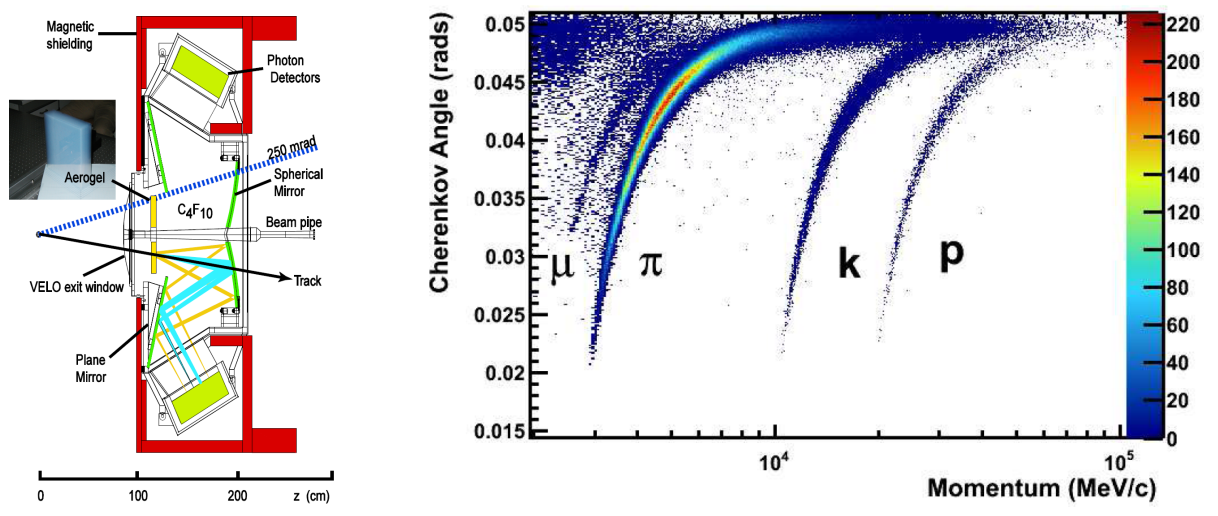

Figure 6: Schematics of the RICH-1 detector of LHCb (left). The two media producing cones are aerogel and $C_{4} F_{10}$. Mirrors extract the light towards the HPD located into magnetic field shielding boxes. The right plot shows the separation among several particle species from $\mathrm{CF}_{4}$ (RICH-2).

Figure 7 demonstrates the efficiency of the RICH to identify particles in the case of B mesons and $\Lambda_{b}$ decays.

Alice has a RICH detector which covers a limited angular acceptance $\left(|\eta|<0.6\right.$ and $1.2^{\circ}<$ $\phi<58.8^{\circ}$ ) and is made from a liquid radiator and gaseous photo-detectors (multiwire proportional chambers, MWPC) to measure the light cone produced.

The performances of the detectors can usually be extracted from the large sample of data produced at LHC and selecting exclusive decays (large branching fraction B decays for example in LHCb [10]) from quantities relying on kinematics only. From those samples, the response of the detector can be precisely determined. 


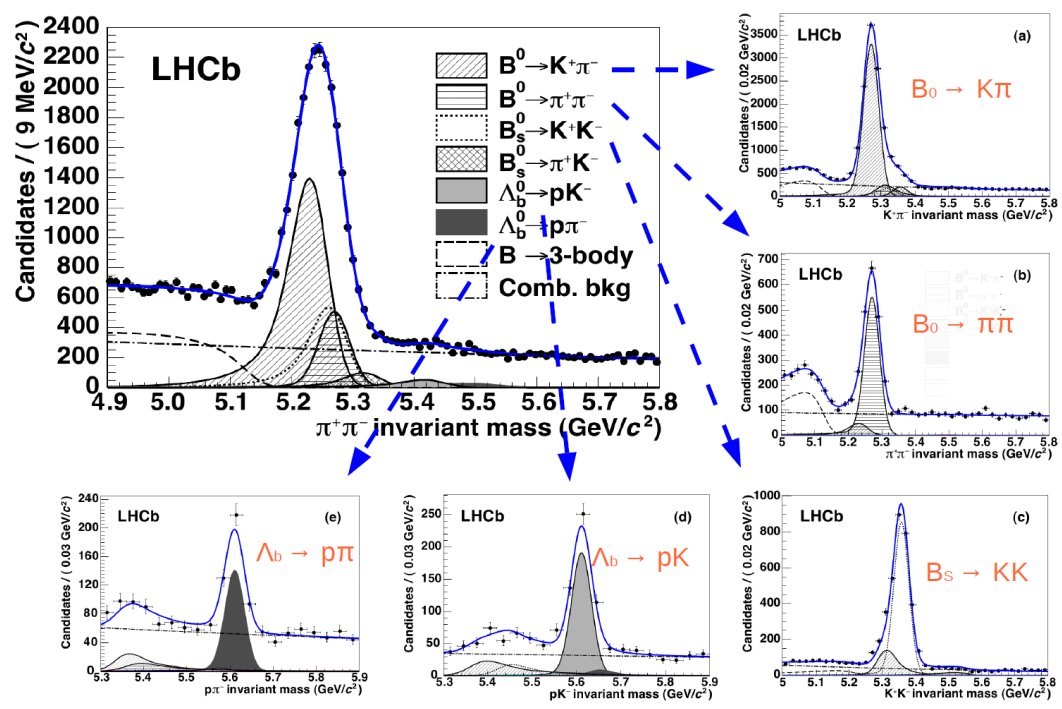

Figure 7: The large top-left plot shows two-body B meson and $\Lambda_{b}$ hadronic decays reconstructed by LHCb when the two particles are interpreted as charged pions; no particle identification is used here. The separation into exclusive decay subsamples ( $B^{0} \rightarrow K \pi(\mathrm{a}), B^{0} \rightarrow \pi \pi$ (b), $B_{s}^{0} \rightarrow K K$ (c), $\Lambda_{b} \rightarrow p K$ (d) and $\Lambda_{b} \rightarrow p \pi$ (e)), based on the RICH identification of the daughter particles leads to the production of sets of candidates with a large efficiency and a low pollution, the mother particle peak being clearly visible.

\subsection{Calorimetry}

The Alice calorimetry is mainly based on the PHOS and EMCAL detectors [11] which are two electromagnetic calorimeters (ECAL). The former is homogeneous and made of $\mathrm{PbWO}_{4}$ crystals read out with avalanche photo-diodes (APD). The angular coverage is limited to $|\eta|<0.13$ and $\Delta \phi \sim 0.6 \pi$ but the detectors benefits from the very good resolution of the technology used: $\frac{\sigma_{E}}{E}=$ $\frac{0.018}{E} \oplus \frac{0.033}{\sqrt{E}} \oplus 0.011(E$ in $\mathrm{GeV})$. The second ECAL is a sampling calorimeter made of layers of lead and scintillator traversed by wavelength shifting fibres (WLS) transporting the light to APD. Although the resolution is more modest, the EMCAL provides a good identification of photons with a readout range up to $250 \mathrm{GeV} / c$ and a far larger angular coverage $\left(|\eta|<0.7\right.$ and $\left.\Delta \phi \sim 110^{\circ}\right)$ (see Fig. 8). Those detectors provide information to the trigger of the experiment.

The electromagnetic calorimeter of LHCb is very similar to the EMCAL. However, the calorimetry in LHCb is based on four sub-detectors [12]: a scintillating pad detector (SPD), a preshower (PRS), the ECAL and a hadronic calorimeter (HCAL) making a pseudo-projective system. The SPD and the PRS are walls of scintillator cells whose light is collected with WLS fibres and read out with multi-anode PMT. A lead wall of $2.5 X_{0}$ inserted between the SPD and the PRS initiates the electromagnetic shower. The ECAL and HCAL follow the PRS on the particle path. The HCAL is a tile detector of 5.6 $\lambda_{I}$ made of scintillator and iron. As for the ECAL, the light is collected by WLS fibres and read out by PMT. A photon produces a significant signal in the PRS and ECAL, electrons in the SPD, PRS and ECAL, charged hadrons in the SPD, potentially ECAL $\left(1.1 \lambda_{I}\right)$ and HCAL and neutral hadrons potentially in ECAL and HCAL. This logic gives to LHCb an already good particle identification at the first trigger level as the calorimeter system is fully read out at $40 \mathrm{MHz}$ altogether with the muon spectrometer. Figure 8 shows the reconstruction of the $B_{s}^{0} \rightarrow \phi \gamma$ 

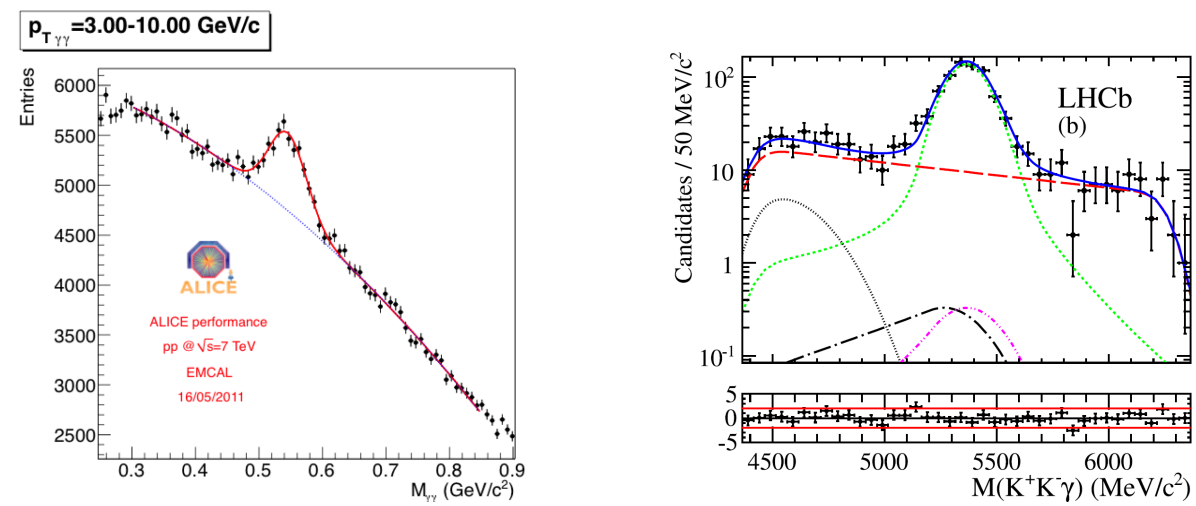

Figure 8: Left: $\gamma \gamma$ invariant mass from the EMCAL calorimeter of Alice and reconstruction of the $\eta$ particle. Right: $B_{s}^{0}$ meson invariant mass reconstruction for the $B_{s}^{0} \rightarrow \phi \gamma$ decay with $\mathrm{LHCb}$ (the green dotted-line is the fitted signal contribution). The resolution (rms width) on the $\mathrm{B}$ is $\sim 92 \mathrm{MeV} / c^{2}$ and is mostly related to the calorimeter resolution.

decays that permitted obtaining the present world best measurement of $\mathscr{B}\left(B_{s}^{0} \rightarrow \phi \gamma\right)[13]$.

\subsection{Muon spectrometers}

Muon identification in LHCb is performed by 5 tracking stations (MWPC, apart from the central part of the first station where triple-GEM technology is used) interleaved with the calorimeter (for the first station) and thick hadron absorbers $\left(\sim 23 \lambda_{I}\right)$ [14]. The trajectories reconstructed in the trackers are extrapolated to the muon spectrometer and a probability for a track to be a muon is determined from the distance of the track with the muon station hits. An efficiency of $97.3 \pm 1.2 \%$ on muon is reached with misidentifications rates $\varepsilon(p \rightarrow \mu) \sim 0.2 \%, \varepsilon(K \rightarrow \mu) \sim 2.4 \%$ and $\varepsilon(\pi \rightarrow \mu) \sim 1.7 \%$ (Fig. 9) [15]. As for the calorimeter, the LHCb muon system is a key ingredient of the first level trigger.

The Alice muon system [16] is also an important device for the experiment trigger. Hence, the Alice experiment implements two types of muon chambers. Seven stations are installed: 5 MWPC and 2 RPC chambers. The formers are used to measure muons offline while the RPC provide a rapid information to the muon trigger of the experiment. The sub-detector is equipped with large absorbers towards the interaction point and to protect the chambers from background incoming from the beam pipe or backwards. A 3 Tm magnet is also installed in between the second and third muon tracking chambers and bends the high momentum muons close to the beam pipe to improve the particle reconstruction.

\section{Conclusion}

The Alice and LHCb experiments have designed, commissioned and run very sophisticated, efficient and reliable detectors for particle identification. Those systems cover a large momentum range which requires the use of many different technologies. This was driven mostly by the specificity of the physics cases of those experiments. The future long shutdowns of the LHC accelerator will be used to further improve the performances of those systems. 


\section{Acknowledgements}

I would like to thank the local organisers for their hospitality, the excellent organisation and the technical support during the conference and the members of Alice and LHCb for their help.

\section{References}

[1] Alice Coll., The Alice experiment at the CERN LHC, JINST 3 (2008) S08002

[2] Alice Coll., Alice : Physics performance report, volume II. J. Phys., G32 (1295-2040), 2006

[3] LHCb coll., The LHCb detector at the LHC, JINST 3 (2008) S08005

[4] LHCb Coll., Implications of LHCb measurements and future prospects, Eur. Phys. J. C 73 (2013)

[5] Alice Coll., The inner tracking system of the Alice experiment, NIM, A530 (28-32), 2004

[6] Alice Coll., The time projection chamber for the Alice experiment, arXiv:0809.5133

[7] Alice TRD Coll., The transition radiation detector for Alice at LHC, Nucl.Phys. A830 (535), 2009

[8] A. Akindinov et al., Performance of the Alice time-of-flight detector at the LHC, Eur. Phys. J. Plus (2013) 128: 44

[9] LHCb Coll., LHCb RICH technical design report, CERN-LHCC-2000-037

[10] M. Adinolfi et al., Performance of the LHCb RICH detector at the LHC, Eur. Phys. J. C73 (2013) 2431

[11] Alice Coll., Alice electromagnetic calorimeter technical design report. 2008, CERN-LHCC-2008-014

[12] LHCb Coll., LHCb calorimeters technical design report, CERN-LHCC-2000-036

[13] LHCb Coll., Measurement of the ratio of branching fractions $\mathscr{B}\left(B^{0} \rightarrow K^{\star 0} \gamma\right) / \mathscr{B}\left(B_{s}^{0} \rightarrow \phi \gamma\right)$ and the direct CP asymmetry in $B^{0} \rightarrow K^{\star 0} \gamma$, Nuclear Physics B, 867 (1)

[14] LHCb Coll., LHCb muon system technical design report, CERN-LHCC-2001-010

[15] A. A. Alves Jr et al., Performance of the LHCb muon system, JINST 8 (2013) P02022

[16] Alice Coll., Alice technical design report of the dimuon forward spectrometer, CERN-LHCC-99-22
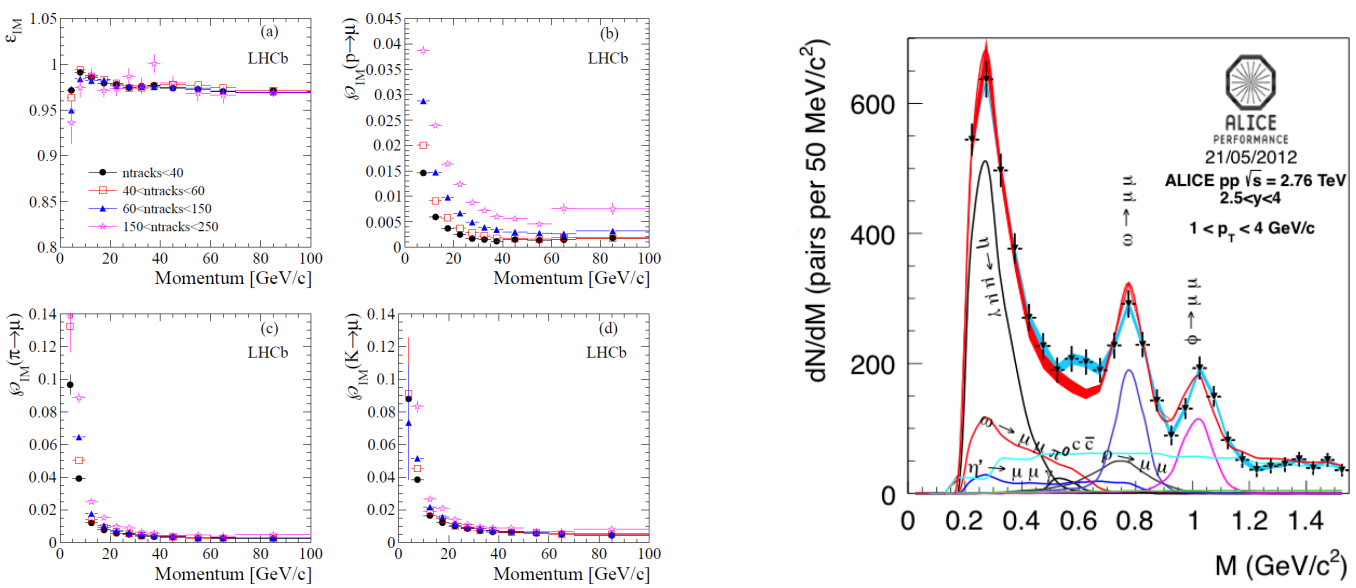

Figure 9: LHCb muon spectrometer efficiency (a) and misidentification rates (b, $c$ and d) as a function of the particle momentum (left). The right plot shows the reconstruction of the invariant mass of resonances built from muon pairs in Alice. 